\title{
Clinical and molecular assessment of regorafenib monotherapy
}

\author{
NAO KAKIZAWA, KOICHI SUZUKI, TARO FUKUI, YUJI TAKAYAMA, KOSUKE ICHIDA, \\ YUTA MUTO, FUMI HASEGAWA, FUMIAKI WATANABE, RINA KIKUGAWA, SHINGO TSUJINAKA, \\ KAZUSHIGE FUTSUHARA, YASUYUKI MIYAKURA, HIROSHI NODA and TOSHIKI RIKIYAMA \\ Department of Surgery, Saitama Medical Center, Jichi Medical University, Omiya-ku, Saitama 330-8503, Japan
}

Received September 5, 2016; Accepted January 30, 2017

DOI: $10.3892 /$ or.2017.5456

\begin{abstract}
Regorafenib has shown survival benefits in metastatic colorectal cancer patients who were exacerbated after all standard therapies. Some patients, however, exhibit severe adverse events (AEs) resulting in treatment discontinuation. Therefore, the selection of patients likely to benefit from regorafenib is crucial. Twenty patients were treated with regorafenib for metastatic colorectal cancer; 122 plasma samples were taken from 16 of these patients for monitoring of circulating tumor DNA (ctDNA) in the blood. The treatment response, AEs, overall survival (OS), progression-free survival (PFS) and tumor morphologic changes on CT images were evaluated. KRAS mutant ctDNA was determined using digital PCR. Median PFS and OS were 2.5 and 5.9 months, respectively. Treatment was discontinued because of disease progression (PD) in 10 patients, and AEs in another 10 patients. AEs included hyperbilirubinemia, severe fatigue and skin rash. Hyperbilirubinemia was seen in two patients with multiple bilateral liver metastases, and severe fatigue in another 2 patients with poor performance status (PS). These severe AEs resulted in treatment discontinuation. Ten patients had a median PFS of 2.1 months with AE related discontinuation; PD occurred at 3.5 months $(\mathrm{P}=0.00334)$. Four patients exhibited a morphologic response, achieving better PFS times of 3.5, 5.3, 5.6 and 14.2 months. Emergence of the KRAS mutation in ctDNA was observed during anti-EGFR antibody treatment in 3 patients among 11 with $K R A S$ wild-type tumors; it was detectable in the blood prior to radiographic detection of PD. Moreover, the KRAS mutation declined in two patients during regorafenib monotherapy. These patients were re-challenged with anti-EGFR antibody. Patients with extensive multiple liver metastases or poor PS are unlikely to benefit from regorafenib. Patients with a morphologic response will probably benefit from regorafenib with adequate management of other AEs. KRAS monitoring in ctDNA could be useful
\end{abstract}

Correspondence to: Dr Koichi Suzuki, Department of Surgery, Saitama Medical Center, Jichi Medical University, 1-847 Amanuma-cho, Omiya-ku, Saitama 330-8503, Japan

E-mail:ksuzbnhm@yahoo.co.jp

Key words: metastatic colorectal cancer, regorafenib, morphologic change, circulating tumor DNA, KRAS mutation regarding treatment response and in determining treatment strategy.

\section{Introduction}

The drug regorafenib is an orally available multi-kinase inhibitor that has shown survival benefits in metastatic colorectal cancer (mCRC) patients who had been exacerbated after all standard therapies $(1,2)$. The CORRECT trial involving regorafenib demonstrated significant survival benefits regarding progression-free survival (PFS) and overall survival (OS) in mCRC patients after the failure of standard therapy (1). The CONCUR trial in 2015 also showed the survival benefits of regorafenib in the Asian population (1). Although significant survival benefits, together with safety in drug management, have been reported in clinical trials, regorafenib is not likely to be used in the clinic because of difficulty in its management. Patients treated with regorafenib have displayed severe adverse events (AEs) such as liver dysfunction and intolerable or hardly manageable fatigue, resulting in discontinuation of the treatment (3-6). These severe AEs would be unlikely to occur in a restricted population, including patients with better performance status in clinical trials, but could happen in clinical practice. Therefore, it is crucial to exclude patients who are likely to exhibit severe AEs and to select patients more likely to benefit from regorafenib monotherapy. For this purpose, clinical and molecular assessment was attempted for the selection of candidates for regorafenib treatment.

The morphologic changes in tumors on enhanced CT images were reported for the first time in 2009 in colorectal cancer patients treated with bevacizumab (7); this drug blocked the activity of vascular endothelial growth factor (VEGF) signaling concerning tumor angiogenesis. Regorafenib also blocks this VEGF signaling via inhibition of the activity in VEGFR1, VEGFR2 and VEGFR3, suggesting that morphologic change could also be induced by regorafenib. Consequently, it could be useful for assessing tumor response and predicting treatment outcome. However, no study has attempted to determine morphologic changes in tumors on CT images during regorafenib monotherapy.

In addition, the dynamic change of genomic profiles was monitored using liquid biopsy in this study. Liquid biopsy is a blood-based technology platform that tracks circulating tumor DNA, allowing multiple testing over time, monitoring of real-time changes within the tumor and evaluation of 
Table I. Morphologic criteria.

\begin{tabular}{llll}
\hline Morphology group & \multicolumn{1}{c}{ Overall attenuation } & Tumor-liver interface & Peripheral rim of enhancement \\
\hline 3 & Heterogeneous & Ill defined & May be present \\
2 & Mixed & Variable & If initially present, partially resolved \\
1 & Homogeneous and hypoattenuating & Sharp & If initially present, completely resolved
\end{tabular}
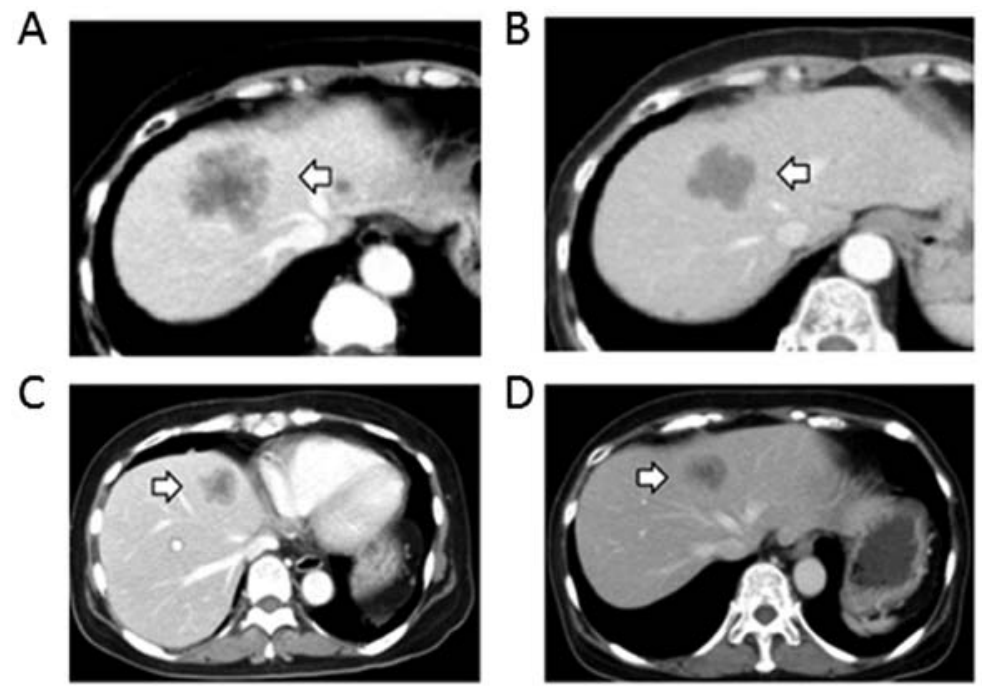

Figure 1. Changes on CT images according to morphologic response. (A and B) Optimal response was defined as the change in morphology from the tumor harboring heterogeneous attenuation with a thick, poorly defined tumor-liver interface (A, group 3) to homogeneous low attenuation with a thin, sharply defined tumor-liver interface (B, group 1). (C and D) No response was defined as the absence of marked changes in morphology (C to D, group 3 to group 3 ).

therapeutic response. There have been several studies that have reported the significance of monitoring of genomic profiles such as EGFR in lung cancer (8) and KRAS in colorectal cancer patients (9-11). In the present study, we monitored $K R A S$ status in circulating tumor DNA (ctDNA), and evaluated its significance concerning molecular assessment for the prediction of treatment response and in aiding decision making in treatment strategy.

\section{Materials and methods}

Patients. Twenty mCRC patients were recruited in the present retrospective study. They underwent regorafenib monotherapy from August in 2013 to January in 2016 at the Saitama Medical Center, Jichi Medical University, Japan. Patients were aged $>18$ years, and their Eastern Cooperative Oncology Group performance status (PS) was 0,1 or 2 . This study was approved by the Research Ethics Committee at Jichi Medical University. Written informed consent was obtained from each study participant.

Assessment. Treatment response, incidence of AEs, PFS, OS and tumor morphologic response on CT images were evaluated. PFS was defined as the time from the start date of this therapy to the first radiological or clinical observation (including elevation of carcinoembryonic antigen) of disease progression (9-11). AEs were graded according to the Common Terminology Criteria for Adverse Events, version 4.0 (CTCAE v4.0). The tumor response or progression was assessed every 3 months using CT or PET-CT using the response evaluation criteria in solid tumors (RECIST) version 1.1, as well as the change in tumor morphology. Tumor morphology was assessed using enhanced CT and characterized according to the criteria previously described (9-11): group 1, homogeneous low attenuation with a thin, sharply defined tumor-liver interface; group 3, heterogeneous attenuation with a thick, poorly defined tumor-liver interface; and group 2, intermediate morphology that could be rated as either group 1 or 3 (Table I). A change in morphology from group 3 or 2 to group 1 was defined as an optimal response (Fig. 1), and a group 3 to group 2 change was defined as an incomplete response. The absence of marked changes in tumor morphology was defined as no response. In patients with multiple tumors, morphologic response was assigned based on the changes observed in the majority of tumors. Response to chemotherapy was also determined using RECIST.

Monitoring of circulating tumor DNA in the blood. During treatment with regorafenib, 122 plasma samples taken from 16 patients were available for the monitoring of ctDNA in the blood. The KRAS status in ctDNA was determined using the droplet digital polymerase chain reaction (ddPCR); this technology provided absolute quantification of DNA with high sensitivity. Seven targets of KRAS mutation including G12D, G12V, G12C, G12R, G12A, G12S and G13D were assessed. Generation of droplets was performed to partition the ddPCR 
Table II. Patient characteristics.

\begin{tabular}{lc}
\hline Patients background & Median (range)/n \\
\hline Age (years) & $67.5(49-76)$ \\
Gender & 13 \\
Male & 7 \\
Female & \\
ECOG PS & 18 \\
$<1$ & 2 \\
2 & \\
Primary site of disease & 9 \\
Colon & 11 \\
Rectum & \\
KRAS status in tumor tissue & 11 \\
Wild-type & 9 \\
Mutant & \\
Number of previous therapies & 1 \\
1 & $80.8(5-43)$ \\
2 &
\end{tabular}

ECOG, Eastern Cooperative Oncology Group; PS, performance status.

reaction mix into thousands of nanoliter-sized droplets. After PCR, droplets from each sample were analyzed individually and read on a well by well basis. The PCR-positive and PCR-negative droplets were counted to provide absolute quantification of the target DNA in digital form. A level of $<0.1 \%$ of positive $K R A S$ mutant circulating tumor DNA was estimated as negative. Amplified products were extracted from the droplets following PCR for Sanger sequencing. To verify the mutation in ctDNA, amplified products were extracted from the droplets following PCR for Sanger sequencing.

Statistical analysis. Fisher's exact test was used to examine the relationship between two categorical variables. Continuous comparisons of variables between two groups were performed. Student's t-test was used to evaluate those variables that followed a normal distribution, and the non-parametric MannWhitney-Wilcoxon test was used for those variables that did not follow a normal distribution. The level of statistical significance was set at $\mathrm{P}<0.05$. Values are shown as the median and range. PFS and OS data were plotted as Kaplan-Meier curves, and the differences among the groups were compared using the log-rank test.

\section{Results}

Patient characteristics. The characteristics of the 20 patients, including 13 males and 7 females, are detailed in Table II. Patient median age was 67.5 (range, 49-76) years. Two patients had a PS of 2 , and the remaining patients had a PS of 0 or 1 .
Table III. Previous treatments.

\begin{tabular}{lc}
\hline Previously used drugs & $\mathrm{n}$ \\
\hline 5-Fluorouracil & 20 \\
Oxaliplatin & 20 \\
Irinotecan & 20 \\
Bevacizumab & 18 \\
Panitumumab/cetuximab & $12^{\mathrm{a}, \mathrm{b}}$
\end{tabular}

${ }^{\mathrm{a}} K R A S$ wild-type $(\mathrm{n}=10)$; ${ }^{\mathrm{b}} K R A S$ mutant in G13D $(\mathrm{n}=2)$.

Table IV. Clinical outcome.

\begin{tabular}{lcc}
\hline Survival & $\begin{array}{c}\text { Median } \\
\text { (months) }\end{array}$ & $\begin{array}{c}\text { Range } \\
\text { (months) }\end{array}$ \\
\hline Progression-free survival & 2.5 & $0.4-14.2$ \\
Overall survival & 5.9 & $1.1-20.9$ \\
\hline
\end{tabular}

Nine patients had colon cancer, and 11 patients had rectal cancer. KRAS analysis of tumor tissue detected no mutation in 11 patients and mutation in 9 patients including 2 patients with mutation of G13D. The median period from diagnosis of metastases before regorafenib monotherapy was 20.8 (range, 4.6-43.0) months. All patients had various previous treatments; single treatment was administered in 1 patient $(5 \%)$, two sequential treatments in $8(40 \%)$ and three sequential treatments in 11 (55\%). Regarding previous treatment, 5-fluorouracil and irinotecan were used in all patients. Oxaliplatin was used in 19 patients (95\%), anti-VEGF antibody and bevacizumab were administered in 18 (90\%), and anti-EGFR antibodies, panitumumab or cetuximab were used in $12(60 \%)$ (Table III). Ten patients $(50 \%)$ received follow on therapy with TAS102.

Treatment exposure. An initial dose of $160 \mathrm{mg}$ was administered in 13 patients. Dose modification in the initial treatment was applied as a result of poor PS or liver damage. Seven patients started with modified initial doses, including 5 with $120 \mathrm{mg}, 1$ with $80 \mathrm{mg}$ and 1 with $40 \mathrm{mg}$. Dose modification during treatment was performed according to the grade of AE. The median relative dose intensity was $76.3 \%$. Dose modification of regorafenib during treatment is shown in Fig. 2.

Efficacy and adverse events. There were no patients with a complete response or a partial response, whereas 3 patients (15\%) showed stable disease. The median PFS and OS times were 2.5 months (range, 0.4-14.2) and 5.9 months (range, 1.1-20.9), respectively (Table III). Treatments were discontinued in all patients. The most common AEs $(\geq 30 \%)$ of any grade were fatigue, hand-foot skin reaction, diarrhea, anorexia, hyperbilirubinemia, anemia and dyspnea. The most common grade 3 or higher $\mathrm{AE}(\geq 20 \%)$ was fatigue (Table IV).

Reasons for treatment discontinuation. The reasons for treatment discontinuation and the duration of administration of 


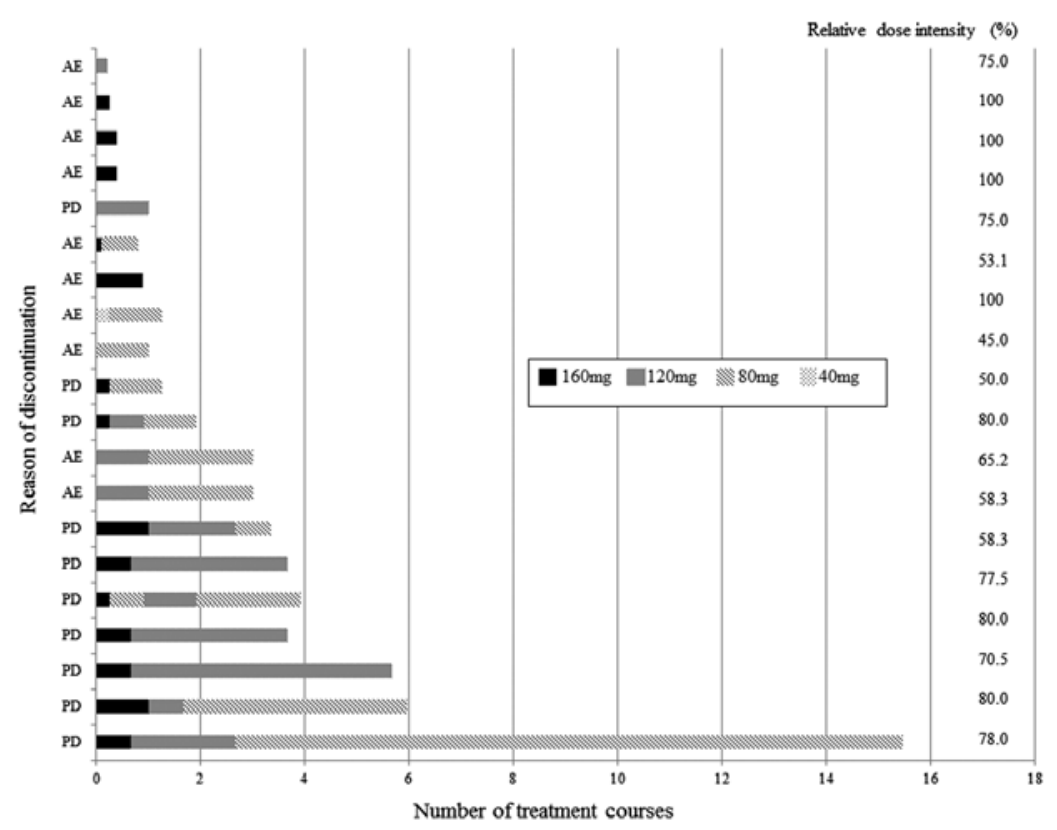

Figure 2. Dose modification of regorafenib during treatment and the reasons for discontinuation. The y-axis shows the reasons for the discontinuation of patients ordered in terms of the number of treatment courses (x-axis). The relative dose of regorafenib in each patient is shown on the right side of the figure. $\mathrm{AE}$, adverse event; PD, progressive disease; black square, $160 \mathrm{mg}$; shaded square, 120 and $80 \mathrm{mg}$; dotted square, $40 \mathrm{mg}$.

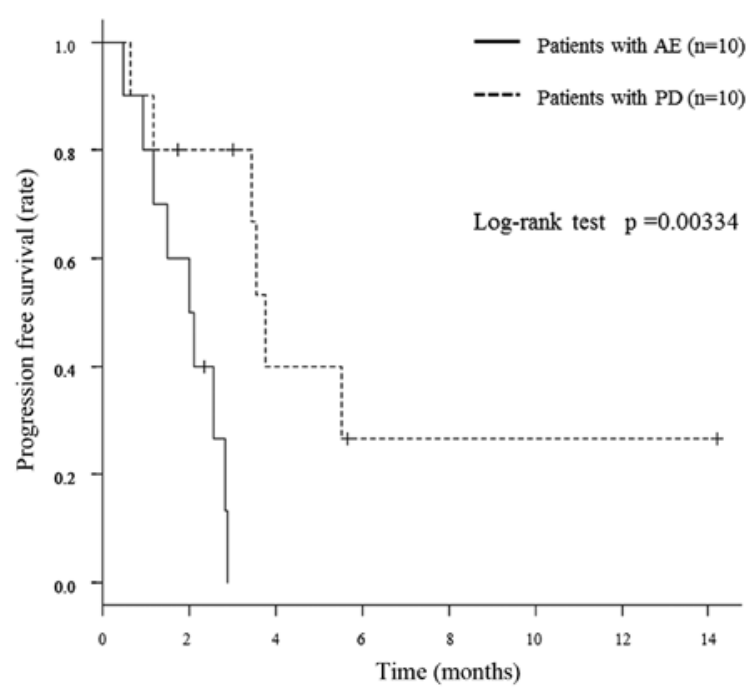

Figure 3. Kaplan-Meier curves showing the comparison of progressionfree survival between patients with treatment discontinuation as a result of adverse events and those caused by progressive disease. AE, adverse event; $\mathrm{PD}$, progressive disease.

regorafenib are shown in Fig. 1. Ten patients (50\%) had progressive disease (PD) and $10(50 \%)$ displayed AEs; the reasons for treatment discontinuation were: ten patients who experienced AEs included 2 with hyperbilirubinemia, 5 with severe fatigue, and 3 with skin rash. Two patients with hyperbilirubinemia had bilateral multiple liver metastases before regorafenib treatment. Two of 5 patients with intolerable or hardly manageable severe fatigue had a PS of 2 before regorafenib treatment. Successful sequential modification was achieved in patients who did not show severe AEs; they had longer treatment duration until discontinuation as a result of PD, while adequate modification failed in patients with severe AEs. Consequently, patients with
Table V. Adverse events.

\begin{tabular}{lcc}
\hline Variables & Any grade (\%) & Grade 3/4 (\%) \\
\hline Clinical adverse events & & \\
Fatigue & 80 & 25 \\
Hand-foot skin reaction & 55 & \\
Diarrhea & 45 & \\
Anorexia & 45 & \\
Voice changes & 15 & \\
Hypertension & 15 & \\
Oral mucositis & 5 & \\
Rash or desquamation & 15 & \\
Nausea & 15 & \\
Weight loss & 20 & \\
Fever & 5 & \\
Constipation & 20 & \\
Dry skin & 25 & \\
Alopecia & 20 & \\
Taste alteration & 20 & \\
Vomiting & 5 & \\
Sensory neuropathy & 10 \\
Dyspnea & 30 & \\
Laboratory abnormalities & \\
Neutropenia & 10 \\
Thrombocytopenia & 20 \\
Hyperbilirubinemia & 40 \\
Proteinuria & 15 \\
Anemia & 35 \\
\hline
\end{tabular}

treatment discontinuation because of PD achieved a significantly better PFS than those with treatment discontinuation as a 
A

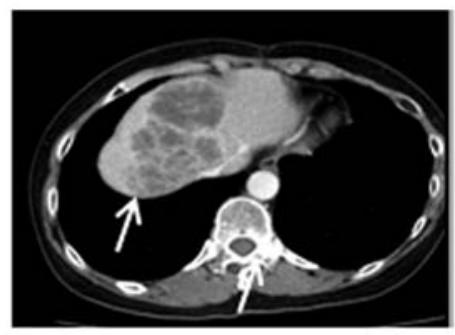

B

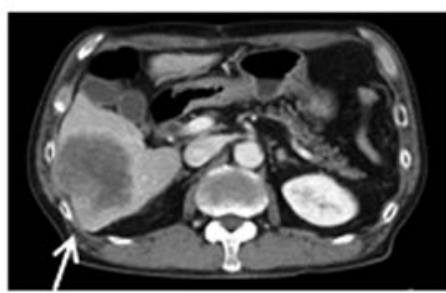

$\mathrm{C}$

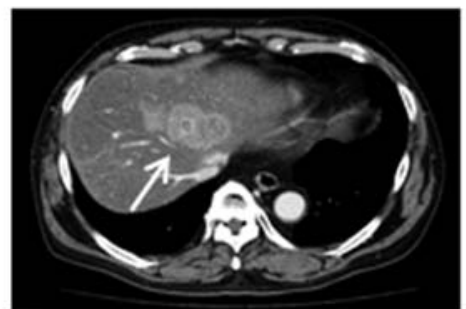

D

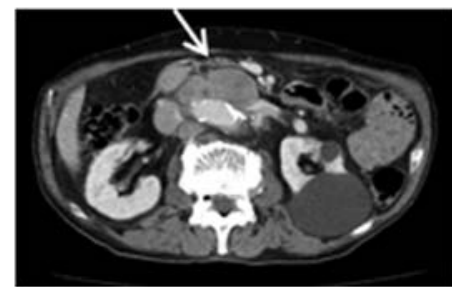

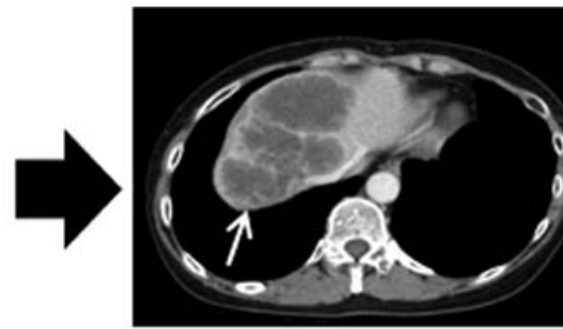
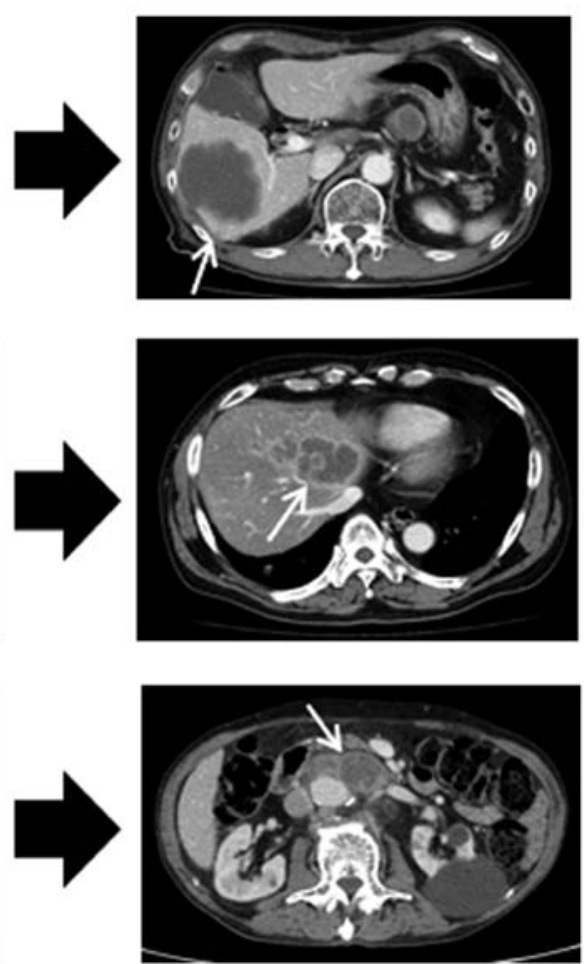

Figure 4. Change in tumor morphology on enhanced CT images before and after treatment with regorafenib. (A) A 50-year-old woman with rectal cancer before (left) and after (right) treatment with regorafenib in 4th line chemotherapy. The change in tumor morphology from heterogeneous to homogeneous low attenuation was seen in the liver metastasis. This patient had PFS of 3.5 months. (B) A 74-year-old man with colon cancer before (left) and after (right) treatment with regorafenib in 4th line chemotherapy. Incomplete response was seen in the liver metastasis. This patient had a PFS time of 5.3 months. (C) A 65 year-old man with rectal cancer before (left) and after (right) treatment with regorafenib in 3rd line chemotherapy. Incomplete response was seen in the liver metastases. This patient had a PFS time of 5.6 months. (D) A 69-year-old man with rectal cancer before (left) and after (right) treatment with regorafenib in 4th line chemotherapy. Incomplete response was seen in the lymph node metastasis. This patient had a PFS time of 14.2 months.

result of AEs (median PFS of 3.5 and 2.1 months, respectively; $\mathrm{P}=0.00334)$, in comparing PFS in terms of discontinuation. Fig. 3 shows the comparison of PFS between patients with treatment discontinuation as a result of PD and those as a result of AEs.

Morphologic changes on enhanced CT. Four patients showed morphologic changes concerning incomplete response on CT images (Fig. 4); in these 4 patients, there were no morphologic changes during bevacizumab treatment. All responses during regorafenib treatment were incomplete; however, the patients with an incomplete response had a longer PFS than the median PFS of 3.5 months in patients who experienced treatment discontinuation because of PD. These changes were detected within 2-3 months from the beginning of regorafenib treatment.

KRAS status in circulating tumor DNA. The emergence of the $K R A S$ mutation in ctDNA was observed in 3 patients among
11 patients without the $K R A S$ mutation in the tumor tissue. Fig. 5 shows the change in KRAS status in ctDNA along with the sequential treatments in these three patients. The emergence of the KRAS mutation in ctDNA was observed during anti-EGFR antibody therapy. The KRAS mutation in ctDNA was detectable in the blood prior to radiographic detection of PD. Moreover, the mutation in ctDNA in 2 out of 3 patients declined during regorafenib treatment. These 2 patients underwent anti-EGFR antibody as re-challenge treatment with the anti-EGFR antibody; 1 patient exhibited the KRAS mutation in ctDNA again after treatments with the anti-EGFR antibody.

\section{Discussion}

The present study revealed that patients with poor PS or multiple bilateral liver metastases are more likely to show hardly manageable severe AEs resulting in the discontinuation of regorafenib monotherapy. These patients displayed poor prognosis with an extremely short PFS of 2.1 months. 


\begin{tabular}{|c|c|c|c|c|c|c|}
\hline $\begin{array}{l}\text { Number of } \\
\text { chemotherapy }\end{array}$ & $1^{\text {st }}$ & $2^{\text {nd }}$ & $3^{\text {nd }}$ & $4^{\text {th }}$ & $5^{\text {th }}$ & $6^{\text {th }}$ \\
\hline Patient 1 & FOLFOX + B & XELIRI+B & Irinotecan $+\mathrm{C}$ & Reg & TAS102 & C \\
\hline Patient 3 & $\mathrm{XELOX}+\mathrm{B}$ & FOLFIRI+C & Reg & TAS1 & Irinotecan $+\mathrm{C}$ & \\
\hline Patient 5 & FOLFOX+B & FOLFIRI+C & Irinotecan $+\mathrm{P}$ & Reg & TAS102 & \\
\hline
\end{tabular}

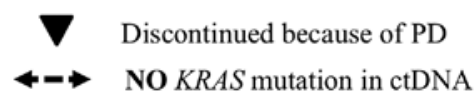

\section{$\nabla \quad$ Discontinued because of AE \\ KRAS mutation in ctDNA}

Figure 5. Emergence of the KRAS mutation in ctDNA in 3 patients without the KRAS mutation in the tumor tissue. Patient 1 is a 50-year-old woman with rectal cancer (the patient shown in Fig. 4A). The KRAS mutation in ctDNA was detected during the 3rd line therapy using irinotecan and cetuximab prior to radiological detection of disease progression (black triangle). Emergence of the KRAS mutation was shown from the dashed arrow to the solid arrow. Moreover, the KRAS mutation in ctDNA disappeared during treatments with regorafenib and TAS102 (from the solid arrow to the dashed arrow). This patient was, therefore, treated with cetuximab as re-challenge treatment with the anti-EGFR antibody. The KRAS mutation in ctDNA was then detected again during the treatment, resulting in discontinuation of treatment because of disease progression. Patient 3 (the patient shown in Fig. 4C) is a 65-year-old man with rectal cancer. KRAS mutation in ctDNA was detected during the 2nd line therapy using FOLFIRI (5-fluorouracil + irinotecan) and cetuximab prior to radiological detection of disease progression. The KRAS mutation in ctDNA disappeared during treatments with regorafenib and TAS102. This patient was being treated with cetuximab as re-challenge treatment with the anti-EGFR antibody. Patient 5 (not shown before) is a 53-year-old woman with colon cancer. The KRAS mutation in ctDNA was detected during the 3rd line therapy using irinotecan and panitumumab prior to radiological detection of disease progression. The $K R A S$ mutation in ctDNA did not disappear during treatments with regorafenib and TAS102, resulting in discontinuation of treatment because of disease progression. The dashed arrow represents the period without KRAS mutation in ctDNA, and the solid arrow represents the period with the KRAS mutation in ctDNA. The black triangle indicates the point at which radiological detection of disease progression was recognized. The white arrow shows the point at which treatment was discontinued because of adverse events. B, bevacizumab; P, panitumumab; C, cetuximab; Reg, regorafenib; FOLFOX, 5-fluorouracil and oxaliplatin; XELOX, capecitabine and oxaliplatin; FOLFIRI, 5-fluorouracil and irinotecan; XELIRI, capecitabine and irinotecan.

In contrast, patients whose tumors exhibited morphological change had a median PFS that was as long as or much longer than 3.5 months. These patients probably benefited from regorafenib; thus, they should receive adequate management and treatment should not be discontinued because of AEs. Furthermore, KRAS monitoring in ctDNA could be useful for the assessment of treatment response and decision making regarding treatment strategy.

Comparison of the profile of AEs in the present study with that in the CORRECT trial revealed that a similar profile in AEs was seen in both. The most common grade 3 or higher AEs were fatigue, skin reaction and hyperbilirubinemia. These AEs were manageable by modification of the dose and/or treatment periods for most of the patients. AEs such as the hand-foot skin reaction, rash and fatigue are most likely to occur during the first or second treatment cycles. Some reports have explored the initiation of regorafenib at a reduced dose as a means of avoiding early toxic effects $(1,2,12)$. In the privious study, successful modification during the early period of treatment was achieved in patients with treatment discontinuation caused by PD. They did not show severe AEs, which resulted in a longer PFS time than that in patients with treatment discontinuation caused by AEs (Figs. 1 and 2). However, severe fatigue and hyperbilirubinemia in patients with a poor PS and multiple bilateral liver metastases, respectively, were hardly manageable and resulted in discontinuation of regorafenib in a short period; these patients were unlikely candidates for regorafenib monotherapy.

In the present study, we attempted to evaluate the morphologic change in tumors on CT images and identify patients likely to benefit from regorafenib. Patients with morphologic changes in their tumors displayed better prognosis with a longer
PFS of $>3$ months. This morphologic change was most likely to occur within 2-3 months of regorafenib treatment; therefore, initial evaluation using CT imaging could provide important information such as treatment outcome in patients likely to benefit from regorafenib. The criteria based on morphologic changes had a significant association with pathologic response and prognosis in mCRC patients with liver metastasis, who underwent chemotherapy including bevacizumab (13). It has been reported that a change in tumor morphology, as determined using CT imaging, presented as vascular reconstruction induced by bevacizumab (14). Tumor morphologic response correctly predicted the pathological changes produced by the antitumor effect of VEGF signaling inhibitor; this indicated that it had predictive value in the prognosis of the patients treated with regorafenib as well as bevacizumab (15). We believed that patients with a better response in the first or second line treatment with bevacizumab would also show better response to treatment with regorafenib, but no association regarding the response was seen. This may have been the result of the difference between the inhibition of VEGF-A in bevacizumab and the multi-kinase inhibitor effect of regorafenib.

The dynamic change in the genomic profiles including $K R A S$ status was monitored in the present study using liquid biopsy. We and other groups $(7,12,15,16)$ have reported that colorectal cancer patients with $K R A S$ wild-type in the tumor display the KRAS mutation in ctDNA during several treatments, including anti-VEGF and EGFR antibody. As we expected, the emergence of the KRAS mutation in ctDNA was observed in three patients during treatment with anti-EGFR antibody. The KRAS mutation in ctDNA in 3 patients was detectable in the blood prior to radiographic detection of PD, suggesting that KRAS monitoring could provide significant 
information concerning drug resistance before routine checkup on CT $(9,10,17)$. Furthermore, we showed for the first time the disappearance of the KRAS mutation in ctDNA in 2 of 3 patients with the $K R A S$ mutation in ctDNA during regorafenib treatment; this indicated the recovery of drug sensitivity to anti-EGFR antibody. These patients underwent re-challenge treatment with the anti-EGFR antibody. For this challenge, regorafenib is the important key drug harboring the power to alter KRAS status with a long period of response. Notably, 1 patient showed $K R A S$ mutation in ctDNA again after treatment with the anti-EGFR antibody. These changes in KRAS status in ctDNA represent the alteration in genomic profile during treatment, suggesting that KRAS monitoring in the blood could be a biomarker not only for treatment response but also in decision making regarding treatment strategy.

In conclusion, our data could provide insights into the clinical value of selecting patients likely to benefit from regorafenib monotherapy. It is important, however, to interpret our results within the context of the study limitations and further studies will be required to draw definitive conclusions.

\section{Acknowledgements}

The present study was supported in part by a grant-in-aid of post graduate students from the Jichi Medical University, a grant-in-aid from the Ministry of Education, Culture, Sports, Science and Technology, and the JKA Foundation through its promotional funds from Keirin Race.

\section{References}

1. Grothey A, Van Cutsem E, Sobrero A, Siena S, Falcone A, Ychou M, Humblet Y, Bouché O, Mineur L, Barone C, et al; CORRECT Study Group: Regorafenib monotherapy for previously treated metastatic colorectal cancer (CORRECT): An international, multicentre, randomised, placebo-controlled, phase 3 trial. Lancet 381: 303-312, 2013.

2. Li J, Qin S, Xu R, Yau TC, Ma B, Pan H, Xu J, Bai Y, Chi Y, Wang L, et al; CONCUR Investigators: Regorafenib plus best supportive care versus placebo plus best supportive care in Asian patients with previously treated metastatic colorectal cancer (CONCUR): A randomised, double-blind, placebo-controlled, phase 3 trial. Lancet Oncol 16: 619-629, 2015.

3. Demetri GD, Reichardt P, Kang YK, Blay JY, Rutkowski P, Gelderblom H, Hohenberger P, Leahy M, von Mehren M, Joensuu H, et al; GRID study investigators: Efficacy and safety of regorafenib for advanced gastrointestinal stromal tumours after failure of imatinib and sunitinib (GRID): An international, multicentre, randomised, placebo-controlled, phase 3 trial. Lancet 381: 295-302, 2013.

4. Funakoshi T, Latif A and Galsky MD: Safety and efficacy of addition of VEGFR and EGFR-family oral small-molecule tyrosine kinase inhibitors to cytotoxic chemotherapy in solid cancers: A systematic review and meta-analysis of randomized controlled trials. Cancer Treat Rev 40: 636-647, 2014.
5. Krishnamoorthy SK, Relias V, Sebastian S, Jayaraman V and Saif MW: Management of regorafenib-related toxicities: A review. Therap Adv Gastroenterol 8: 285-297, 2015.

6. McLellan B, Ciardiello F, Lacouture ME, Segaert S and Van Cutsem E: Regorafenib-associated hand-foot skin reaction: practical advice on diagnosis, prevention, and management. Ann Oncol 26: 2017-2026, 2015.

7. Chun YS, Vauthey JN, Boonsirikamchai P, Maru DM, Kopetz S, Palavecino M, Curley SA, Abdalla EK, Kaur H, Charnsangavej C, et al: Association of computed tomography morphologic criteria with pathologic response and survival in patients treated with bevacizumab for colorectal liver metastases. JAMA 302: 2338-2344, 2009.

8. Maheswaran S, Sequist LV, Nagrath S, Ulkus L, Brannigan B, Collura CV, Inserra E, Diederichs S, Iafrate AJ, Bell DW, et al: Detection of mutations in EGFR in circulating lung-cancer cells. N Engl J Med 359: 366-377, 2008.

9. Diaz LA Jr, Williams RT, Wu J, Kinde I, Hecht JR, Berlin J, Allen B, Bozic I, Reiter JG, Nowak MA, et al: The molecular evolution of acquired resistance to targeted EGFR blockade in colorectal cancers. Nature 486: 537-540, 2012.

10. Misale S, Yaeger R, Hobor S, Scala E, Janakiraman M, Liska D, Valtorta E, Schiavo R, Buscarino M, Siravegna G, et al: Emergence of KRAS mutations and acquired resistance to anti-EGFR therapy in colorectal cancer. Nature 486: 532-536, 2012.

11. Siravegna G, Mussolin B, Buscarino M, Corti G, Cassingena A, Crisafulli G, Ponzetti A, Cremolini C, Amatu A, Lauricella C, et al: Clonal evolution and resistance to EGFR blockade in the blood of colorectal cancer patients. Nat Med 21: 795-801, 2015.

12. Tabchi S and Ghosn M: Regorafenib: Start low and go slow. Target Oncol 10: 445-447, 2015.

13. Klinger M, Tamandl D, Eipeldauer S, Hacker S, Herberger B, Kaczirek K, Dorfmeister M, Gruenberger B and T: Bevacizumab improves pathological response of colorectal cancer liver metastases treated with XELOX/FOLFOX. Ann Surg Oncol 17: 2059-2065, 2010.

14. O'Connor JP, Carano RA, Clamp AR, Ross J, Ho CC, Jackson A, Parker GJ, Rose CJ, Peale FV, Friesenhahn M, et al: Quantifying antivascular effects of monoclonal antibodies to vascular endothelial growth factor: insights from imaging. Clin Cancer Res 15: 6674-6682, 2009.

15. Suzuki K, Muto Y, Ichida K, Fukui T, Takayama Y, Kakizawa N, Kato T, Hasegawa F, Watanabe F, Kaneda Y, et al: Morphologic response contributes to patient selection for rescue liver resection of chemotherapy patients with initially unresectable colorectal liver metastasis. Oncol Lett (In press).

16. Mross K, Frost A, Steinbild S, Hedbom S, Buchert M, Fasol U, Unger C, Kratzschmar J, Heinig R, Boix O, et al: A phase I doseescalation study of regorafenib (BAY 73-4506), an inhibitor of oncogenic, angiogenic, and stromal kinases, in patients with advanced solid tumors. Clin Cancer Res 18: 2658-2667, 2012.

17. Nielsen DL, Palshof JA, Larsen FO, Jensen BV and Pfeiffer P: A systematic review of salvage therapy to patients with metastatic colorectal cancer previously treated with fluorouracil, oxaliplatin and irinotecan +/- targeted therapy. Cancer Treat Rev 40: 701-715, 2014. 